$5^{\text {th }}$ International Conference on Innovation in Science and Technology

Barcelona - Spain

ISTCONF

7 - 9 December, 2018

\title{
FORMULATION AND ANTIOXIDANT ACTIVITY OF A CREAM CONTAINING VITIS VINIFERA.L LEAF EXTRACT
}

\author{
A. Chenafa ${ }^{1,2^{*}}$, M.A. Selka ${ }^{1,2}$, A. Boulerial ${ }^{1}$, B. Talha ${ }^{1}$, H. Toumi ${ }^{2}$ \\ ${ }^{1}$ Department of Pharmacy, Djillali Liabes University of Sidi-Bel-Abbes, Algeria \\ ${ }^{2}$ Laboratory of Research in Pharmaceutical Development, Oran, Algeria
}

\begin{abstract}
Red vine (Vitis vinifera L.) is a plant easily procurable in Algeria, rich in various polyphenols (flavonoid, anthocyanin...) at various parts, yet it is still poorly exploited. These polyphenols have numerous properties including antioxidant property.

As a part of the valuation of the Algerian flora, the aim of this study was to formulate a cream containing an extract of red vine leaves and evaluate its antioxidant potential.

The extract was obtained by hydro-alcoholic extraction of Vitis vinifera.L leaves. Two creams "O/W" were prepared (base-formulation). The stability of these creams was studied at two different temperatures $25^{\circ} \mathrm{C}$ and $40^{\circ} \mathrm{C}$ for 60 days.

Creams were physicochemically stable. The study of antioxidant activity by the method of free radical scavenging using DPPH (2,2- diphenyl- 1 - picrylhydrazyl ) and the comparison with the two antioxidants of reference ( ascorbic acid and Butylhydroxytoluene ) revealed a moderate antioxidant potential for the extracts as well as the creams but with a minor potential.

In vivo studies are recommended to confirm the antioxidant action of such creams as well as the study of its long- term stability.
\end{abstract}

Keywords - antioxidant potential, emulsion, stability, Vitis vinifera L.

\section{Themes: Sciences}

